Journal of Engineering and Applied Sciences 15 (3): 819-825, 2020

ISSN: 1816-949X

(C) Medwell Journals, 2020

\title{
Pose Effect Analysis with Accuracy of Archery using Manhattan Distance Algorithm
}

\author{
Lutfi Handi Saptiawan, Tito Waluyo Purboyo and Anton Siswo Raharjo Ansor \\ Department of Computer Engineering, Faculty of Electrical Engineering, \\ Telkom University, Bandung, Indonesia
}

\begin{abstract}
In archery basic techniques there are steps that need to be taken into account from starting to stand up to resisting archery or after hold. The use of this basic technique is one of the keys in archery sports. This sport itself uses memory muscle, so that, beginners and professional archers do not escape the mistakes when shooting that often occur due to changes in the attitude of the archers themselves. In this study, a search for archery athlete's body parts will tend to change when shooting from the aiming position to after hold, using a motion capture tool and using the Manhattan distance calculation method to find deviation values in several body parts.
\end{abstract}

Key words: Archery, motion capture, Manhattan distance, calculation, technique, shooting

\section{INTRODUCTION}

In an archery sport competition, participants collect points as high as possible to win the match. Therefore, getting the highest score becomes an important point in a match or championship in archery (Kolayis et al., 2014). There are various kinds of factors as well as the basic things that affect archery sports to be able to obtain high scores including there are arc factors that are used and techniques used in making shots that will affect the precision in regarding the target (Guest and Walls, 2017) therefore, the technique or pose in archery is one of the, factors to get good shot precision (Vasquez, 2017; Adams, 2018).

Motion capture is a term in a method of recording a person's movements in the form of three-dimensional data to be developed as well as filmmaking, animation, 3D games or analysis of the data obtained. From the results of analysis of data that has been processed from motion capture devices, it can be concluded that which body parts have the highest deviation value from the archer when shooting from aiming poses to releasing arrows.

\section{Literature review}

Archery: In the process of archery, there are several basic techniques, namely, Stance, Nocking the Arrow, Set, Set-up, Draw and Load, Anchor, Transfer and Hold, Aim and Expand, Release, Follow-Through. And data recording will be carried out during the stance until a release is made.

Motion capture: Motion capture is a technology that serves to record a movement into three-dimensional digital data to be developed or analyzed
(Estevez-Garcia et al., 2015; Sharma et al., 2013). In this study, a method of motion capture device is used in the type of non-optical motion capture where this type of model can be used to record movement data that is relatively simple, so that, it meets the standards in archer motion data retrieval.

Manhattan distance: Manhattan distance is a method that has the same function as Euclidean distance and an algorithm associated with calculating the distance between two points (Ponnmoli and Selvamuthukumaran, 2014). Manhattan distance calculation method can be applied in conducting pattern matching/recognition as well as recognition in an figure which is a second dimension data (Ponnmoli and Selvamuthukumaran, 2014; Khosla et al., 2014; Aprilia et al., 2018) and used in grouping data or commonly known as classification data using the K-method. Nearest neightbor with Manhattan distance as a supporting mechanism (Hammam et al., 2017; Sinwar and Kaushik, 2014). Study of Euclidean and). The mathematical formula of Manhattan Distance (MD) de ned as:

$$
\operatorname{MD}(\mathrm{c} 1, \mathrm{c} 2)=\sum_{\mathrm{i}=1}^{\mathrm{n}}\left|\mathrm{c} 1_{\mathrm{i}}-\mathrm{c} 2_{\mathrm{i}}\right|
$$

Where:

$\begin{array}{lll}\text { MD (ci, c2) } & \text { Manhattan Distance (c1, c2) } \\ \mathrm{c1}_{\mathrm{i}} & : \text { Coordinate } 1 \text { of } \mathrm{i} \\ \mathrm{c} 2_{\mathrm{i}} & : & \text { Coordinate } 2 \text { of } \mathrm{i} \\ \mathrm{i} & : & \text { Dimension } \\ \mathrm{n} & : & \text { Amount of data }\end{array}$

Same as $\mathrm{ED}$ where, $\mathrm{c} 1_{\mathrm{i}}$ and $\mathrm{c} 2_{\mathrm{i}}$ is the values coordinate and are amount of data or dimension. 


\section{Related format file}

Biovision hierarchical file: $\mathrm{BHV}$ is a format by adding skeleton-hierarchy into motion data, so that, it contains many data hierarchical frameworks. BVH is a commonly used format compared to other motion capture formats (Meredith and Maddock, 2001).

Comma separated values file: Comma separated values is a file with landscape data format that contains values with comma ',' or semicolons ';' as separators between values (Umbrich et al., 2015) and this format is also commonly used in making it easy to enter data into a data base simply.

Open Office: With the same functions as Microsoft Office, Open Office has several features such as word processing, worksheets and data bases.

Microsoft Excel: Microsoft Excel is a spreadsheet program that is software that is used to handle process data in the form of rows and columns easily (Held et al., 2018).

\section{MATERIALS AND METHODS}

Collecting data: First of all, we need to install and configurate the application of perception neuron. The Perception Neuron application must installed to connect the perception neuron hardware to the laptop. Neuron perception provides an application version for 32 bits and 64 bits and in this study 64-bit versions will be used, so that, the program's performance will work better. Installation of equipment in athletes is carried out after the installation stage of the program has been completed. The perception neuron device as a whole has 17 sensors that can be used carried out after all the sensors in the perception neuron tool have been detected by the program. This is done to reduce the possibility of loss of contact between the sensor and the Perception Neuron program or to avoid a zero rotation error in the sensor, so that, the calibration process must be carried out every time a motion is recorded.

Converting: Data conversion is intended to convert raw data from neuron perception applications into data that can be read and can be processed for analysis. The process of data conversion itself has stages to obtain the desired data, namely:

Converting data to BVH file format: $\mathrm{BVH}$ is the format used to store motion capture data. Converting data into $\mathrm{BVH}$ format can be done using the export menu on the Axis Neuron program menu before export. The frame to be taken starts from the movement holding back the archery until the release attitude.
Converting data to CSV: CSV is a data processing file in landscape form that uses commas or semicolons as data separators (Umbrich et al., 2015). In doing conversion, use Microsoft Office Word applications and Open Office Writer. Microsoft Office Word is used to open BVH files, then all contents in Microsoft Office Word are copied into Open Office Wirter. This is done because Microsoft Office Word supports opening BVH but it cannot save as text encoded, so, it needs to be copied into Open Office Writer to be stored as text encoded. Data in CSV is opened using Open Office, so that, the format of each column and row does not change and then some of the data needed is copied into Microsoft Excel. This is because Microsoft Office Excel is a spreadsheet program that can be used to process data in the form of rows and columns efficiently, so that, conversion is needed in the form of Excel.

Search for deviation values: In the search for deviation values based on each athlete pose carried out using Manhattan distance calculations using the MATLAB program. The program flowchart in MATLAB in finding the deviation value can be seen in Fig. 1.

The program that has been created using MATLAB starts from giving indigo to common variables and reading the contents of each Excel data to be moved into the array string variable in MATLAB. To be able to find out from the name of the part or part of the sensor, also read the name of the sheet in the Excel file using the functions that are in MATLAB. Then the name that has been read will be entered into the variable used to rewrite when it will create a new Excel file as the output of the MATLAB program.

After the data to be used has been moved into the MATLAB database, it is read and searched for the average value of each frame. The reading of data values is carried out on all sheet excel representing the $\mathrm{x}, \mathrm{y}$ and $\mathrm{z}$ axes. After reading the score and getting the average value of each $\mathrm{x}, \mathrm{y}$ and $\mathrm{z}$ axis on each sheet in each Excel file, a score index that has the highest score is performed. The highest score will be the main value in determining the pose that is considered right. With the highest value on archery being a bullseye with a value of 10 in this study the bullseye value will be made to 11 to distinguish between bullseye and 10 points. However, it is possible that the archer or athlete managed to fire more than once with a bullseye score (idx_max $>1$ ), so that, making only one pose as a reference value while there are more than one pose that is considered right will be the wrong thing. So, it is necessary to search for the average return value for the highest suspended pose.

After obtaining the average value of the highest suspended pose, then the deviation value search process can be carried out using the highest suspended pose data as a reference. The following is the source code used to melt the deviation value of each part in the pose. 


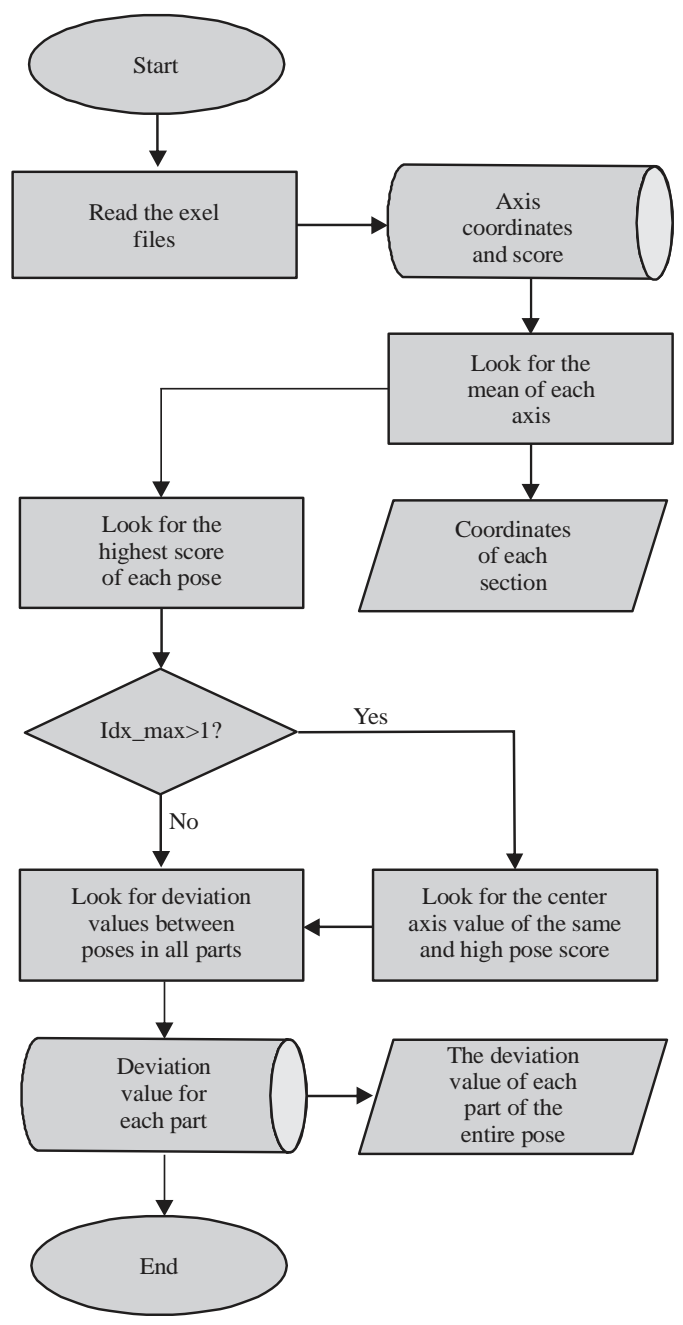

Fig. 1: Flowchart program

$$
\begin{aligned}
& \text { for } p=1 \text { :bp } \\
& \text { fors }=1 \text { :numel(nama_sheet) } \\
& \mathrm{N}=0 \text {; } \\
& \text { foraxis }=1 \text { :bs } \\
& \text { distance }\{\text { sumbu }\}=\text { abs } \\
& \text { (file }\{\mathrm{bp}+1 \text {,s,axis }\} \text {-file }\{\mathrm{p}, \mathrm{s}, \mathrm{axis}\} \text { ); } \\
& \mathrm{N}=\mathrm{N}+\text { distance }\{\text { sumbu }\} \text {; } \\
& \text { end } \\
& \text { ex_pose_sheet }\{\mathrm{p}, \mathrm{s}\}=\mathrm{N} \text {; } \\
& \text { ex_sheet }\{\mathrm{s}\}=\mathrm{N} \text {; } \\
& \text { end } \\
& \mathrm{M}=0 \text {; } \\
& \text { fors }=1 \text { :numel(nama_sheet) } \\
& \mathrm{M}=\mathrm{M}+\text { ex_sheet }\{\mathrm{s}\} \text {; }
\end{aligned}
$$

Based on the source code above, $p$ is the number of Excel files is the number of sheets or parts of the sensor, axis is the number of coordinates, $\mathrm{N}$ is the deviation value per part of ex_pose_sheet, while $\mathrm{M}$ is the accumulation of all the deviations of each part. After obtaining a deviation value from each part of the pose and total deviation. It is necessary to re-search the average value of each part of the pose or sheet based on the score grouping.

\section{RESULTS AND DISCUSSION}

Based on the coordinates of the raw data of the movement of three archers who have been converted and carried out a thorough calculation using the MATLAB program, the program output or output is in the form of Excel data containing the sequence of shots, shot points, deviations per section, total deviation and graphs in the form of visualization of the values in Excel.

First archer: In the first archer, the distance between the target and the archer is $20 \mathrm{~m}$ long and $170 \mathrm{~cm}$ high and the pull length is 26-28 inches. Archers shoot 30 times with 1 session having 6 shots. There are five poses with different scores namely poses with scores of ten, nine, eight, seven and six. The following is the table of pose parts with the highest average deviation based on the score of the shot score.

Based on Table 1, it can be seen that the pose with a score of 8 has right foot, left up leg and left foot deviation values but the left hand part becomes the smallest value of all poses with a value of 0.341459 , so that, it is stable on the target path even though the deviation value is get big because of the three parts above. The following is a ploting of the first archer's left hand.

In Fig. 2, it is a plotting figure from the position of the right hand when performing the stance until the release of the shot. The figure was taken from the corner over the Northeastern direction of the archer. The colored dot in the figure is the location of the sensor that represents the part of the archer's body that is connected to the blue line, so, it makes a skleton pattern. From Fig. 2, the part that has a lot of position changes is in the right hand part that is connected to the left forearm and left arm position. At the left hand point dark blue in the figure is the highest score which is worth the bullseye. The most distorted direction in Fig. 2 shows a line with a bright blue dot that is worth a score of 6 . Shots in score 6 tend to be more towards the right, while shots with a bullseye score are more likely to the left, so that, the first archer looks to the right. Below is a ploting of the right hand of the first archer.

In Fig. 3 is a plotting of the right hand part archer, a figure taken from the northeast corner of the archer. Same as Fig. 2 and 3 is recorded from the stance movement, aiming to release. Changing the position of the right hand every shot from the first archer 
J. Eng. Applied Sci., 15 (3): 819-825, 2020

Table 1: Value of the first archer's average deviation

\begin{tabular}{lcccccc} 
Total distance & Point & Right foot & Left foot & Right shoulder & Right arm & Right forearm \\
\hline 11.62434 & 10 & 4.032583 & 3.895847 & 0.169918 & 0.16578 & 0.888600 \\
7.386571 & 9 & 2.439023 & 2.122245 & 0.163540 & 0.306558 & 0.419318 \\
47.16893 & 8 & 11.380160 & 11.801390 & 3.051398 & 2.651666 & 5.388271 \\
9.297534 & 7 & 2.602859 & 2.950119 & 0.155638 & 0.153624 & 1.001583 \\
5.492914 & 6 & 1.397419 & 0.957951 & 0.129231 & 0.581934 & 0.522685 \\
\hline Total distance & Point & Right hand & Left shoulder & Left arm & Left forearm & Left hand \\
\hline 11.62434 & 10 & 1.057433 & 0.049314 & 0.138846 & 0.289343 & 0.394596 \\
7.386571 & 9 & 0.600975 & 0.045803 & 0.072335 & 0.216644 & 0.446226 \\
47.16893 & 8 & 0.672692 & 0.028230 & 0.061211 & 0.241628 & 0.341459 \\
9.297534 & 7 & 1.061368 & 0.023874 & 0.076758 & 0.330707 & 0.452665 \\
5.492914 & 6 & 0.630903 & 0.036694 & 0.074592 & 0.24314 & 0.476428 \\
\hline
\end{tabular}

Table 2: Value of the second archer's average deviation

\begin{tabular}{lcccccc}
\hline Total distance & Point & Right foot & Left foot & Right shoulder & Right arm & Right forearm \\
\hline 4.583937 & 10 & 2.241553 & 1.334561 & 0.041747 & 0.036080 & 0.065845 \\
9.848485 & 9 & 4.177224 & 4.337090 & 0.085129 & 0.053093 & 0.100547 \\
5.282591 & 8 & 1.820305 & 1.722297 & 0.086692 & 0.097427 & 0.136599 \\
5.997822 & 7 & 2.172463 & 2.080488 & 0.045640 & 0.082201 & 0.115981 \\
7.979959 & 6 & 3.031926 & 3.183645 & 0.065389 & 0.143574 & 0.154313 \\
7.580344 & 5 & 3.224002 & 2.935808 & 0.086263 & 0.080216 & 0.103495 \\
13.21807 & 4 & 5.138817 & 5.840404 & 0.041980 & 0.277554 & 0.247500 \\
\hline Total distance & Point & Right hand & Left shoulder & Left arm & Left forearm & Left hand \\
\hline 4.583937 & 10 & 0.099383 & 0.050147 & 0.035346 & 0.072075 & 0.281884 \\
9.848485 & 9 & 0.238482 & 0.061570 & 0.072499 & 0.157522 & 0.141225 \\
5.282591 & 8 & 0.278458 & 0.070160 & 0.121584 & 0.127068 & 0.347680 \\
5.997822 & 7 & 0.219863 & 0.061554 & 0.116792 & 0.136438 & 0.471811 \\
7.979959 & 6 & 0.159998 & 0.061918 & 0.050289 & 0.284373 & 0.357081 \\
7.580344 & 5 & 0.172301 & 0.056024 & 0.093705 & 0.057125 & 0.325591 \\
13.21807 & 4 & 0.267016 & 0.039941 & 0.252940 & 0.277056 & 0.382525 \\
\hline
\end{tabular}

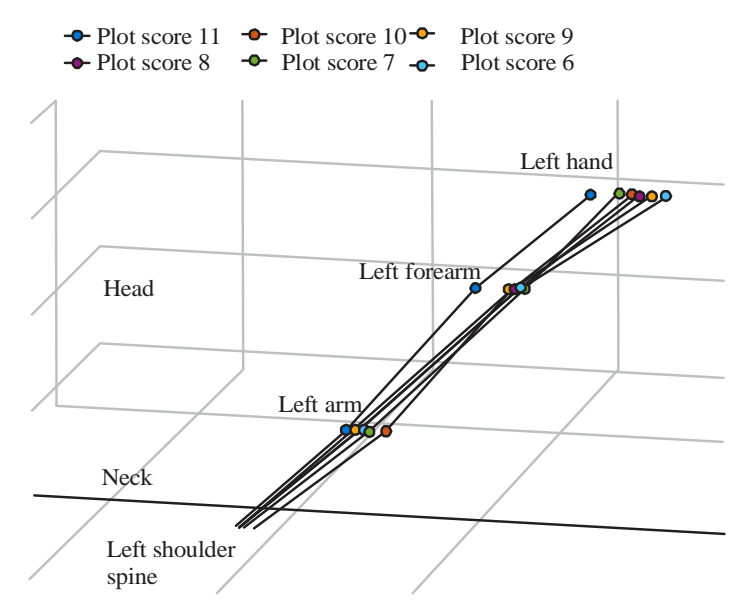

Fig. 2: Plotting the first archer's left hand

is very significant, this can be seen from the position of the color point in Fig. 3. The color point in the figure represents the body part of the archer connected to the blue line, so that, skeleton pattern is specifically, right hand part. Based on Fig. 3 above, right hand with a bullseye score in dark blue tends to be more parallel when compared to the right hand of the other scores. At a score of 10 , even though it is located far enough based on Fig. 3 above but the position is still more parallel than the score of 6 .

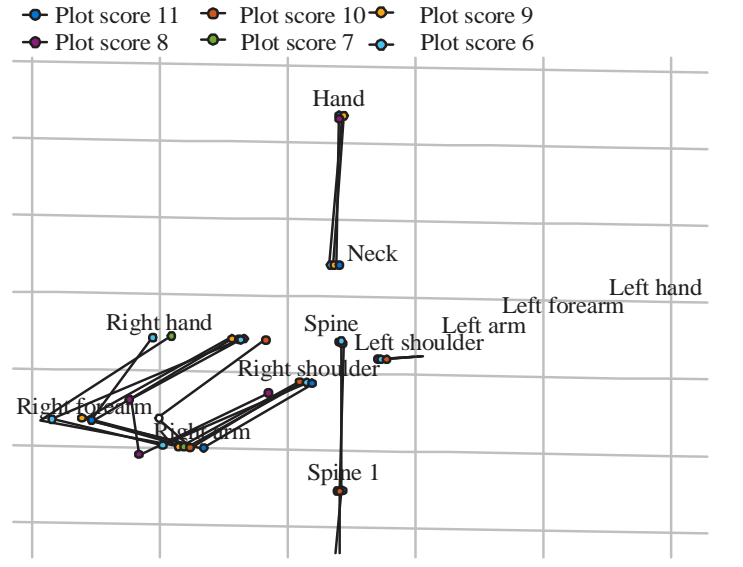

Fig. 3: Plotting the first archer’s right hand

Second archer: In the second archer, the distance between the target and the archer is $18 \mathrm{~m}$ long with $155 \mathrm{~cm}$ height and the pull length is 24 inches to 26 inches. Archers shoot 42 times with 1 session having 6 shots. The following is the table of pose parts with the highest average deviation based on the score of the shot score.

Based on Table 2, the total deviation value is sequential from the highest score which has the smallest total deviation value to score 4 which represents the miss value. At the score of nine, the average deviation value is 
J. Eng. Applied Sci., 15 (3): 819-825, 2020

Table 3: Value of the second archer's average deviation

\begin{tabular}{lclllcc}
\hline Total distance & Point & Right foot & Left foot & Right shoulder & Right arm & Right forearm \\
\hline 4.454384 & 10 & 1.358506 & 1.662052 & 0.037257 & 0.079811 & 0.16625 \\
7.468311 & 9 & 2.185508 & 2.487032 & 0.076684 & 0.158273 & 0.546147 \\
6.968434 & 8 & 1.920614 & 2.864817 & 0.057483 & 0.129103 & 0.322847 \\
\hline Total distance & Point & Right hand & Left shoulder & Left arm & Left forearm & Left hand \\
\hline 4.454384 & 10 & 0.184313 & 0.061229 & 0.150359 & 0.095109 & 0.269107 \\
7.468311 & 9 & 0.524795 & 0.048160 & 0.067801 & 0.275585 & 0.619423 \\
6.968434 & 8 & 0.380806 & 0.033633 & 0.074677 & 0.278775 & 0.340459 \\
\hline
\end{tabular}

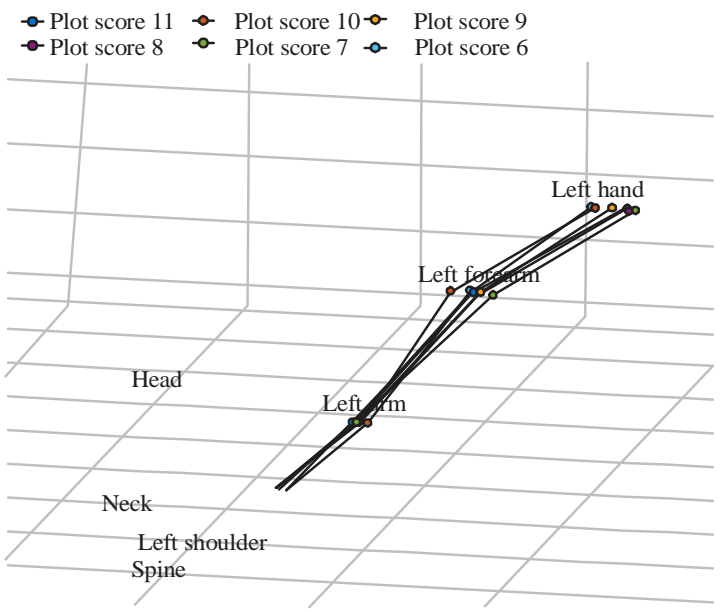

Fig. 4: Plotting the second archer's left hand

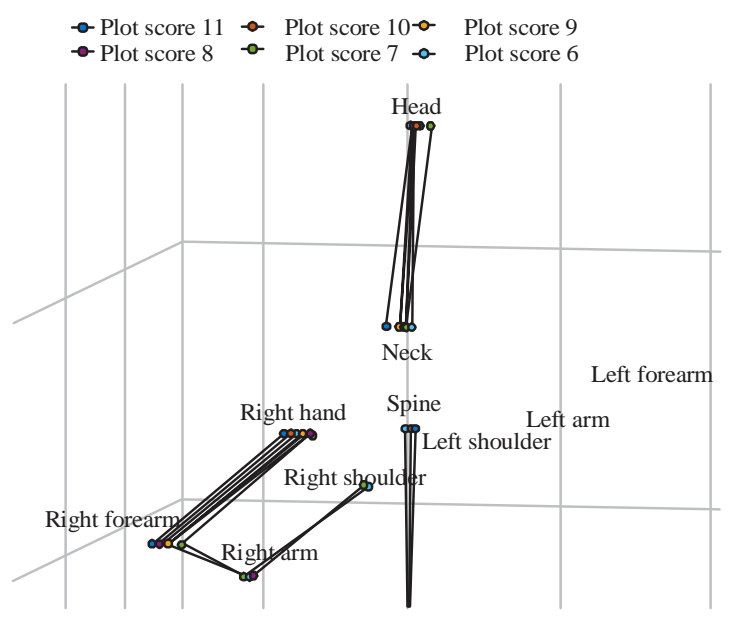

Fig. 5: Plotting the second archer’s right hand

higher than the score after 8,7 and 6 . However, the deviation value at the score of nine is smaller, so that, the shot can be interpreted in the direction of the target or approaching the pose with the highest score. The following is the ploting of the second archer's left hand.

Same as Fig. 2, Fig. 4 above is a pattern of a left-handed skeleton, the pattern of the skeleton is seen from the Northeast over the second archer with a focus on the left hand. The point in the figure is also a sensor that represents the left hand part of the second archer. In Fig. 4 above is a figure of the position of the second right hand archer. You can see the position with a score of 10 seen more towards the left from the left forearm position. At scores 7, 6 and 5 have almost the same position which tends to go to the right from the left hand position score of 10 , so that, when the match misses the target. the direction of the second archer's shot is more likely to the right. The following is a ploting from the right hand of the second archer.

Same as Fig. 3, Fig. 5 is also a plotting figure of the right hand part of the second archer, the figure is taken from the Northeast corner of the archer. The color point in the figure is the location of the sensor that represents the body part of the archer which is connected to the blue line, so as to make skeleton, especially, the right hand part. In Fig. 5, the change in the point pattern or pose of the left hand above is much more regular when compared to the change of the first archer's right hand in Fig. 3. The right hand position in yellow tends to be towards the left. Whereas other positions with a distance that is quite far from the right hand position the score of 10 which is a score of 6 and a score of 7.

Third archer: In the third archer, the distance between the target and archers is $18 \mathrm{~m}$ long with $160 \mathrm{~cm}$ height and 26-28 inches of pull length. Archers shoot 14 times with 1 session having 6 shots. The following is the table of pose parts with the highest average deviation based on the score of the shot score.

Based on Table 3, there are only three scores that are obtained, namely ten, nine and eight. Pose with a score of 10 has the smallest total deviation while the value of nine has a greater deviation value than the pose score of eight. The following is a figure of plotting the left hand.

As shown in Fig. 2 and 4, Fig. 6 is the position of the skeleton of the third archer of the left hand. Capturing a figure of a skeleton from the northeast of the archer. The color points in Fig. 6 are part of the sensor from the left hand because the score obtained by the third archer has only three types, so, the observation is much easier to do. In Fig. 6 because the highest score is based on the value of the average score of 10 , so that, it has a parallel position. Position 10 tends to be straight with the position of score 8 because the position on the right hand score 9 tends to point to the left, so that, it can be said because the smallest score is in the direction that tends to the right, the archer will be more likely to miss the right target. Here, is a ploting from the third archer's right hand. 


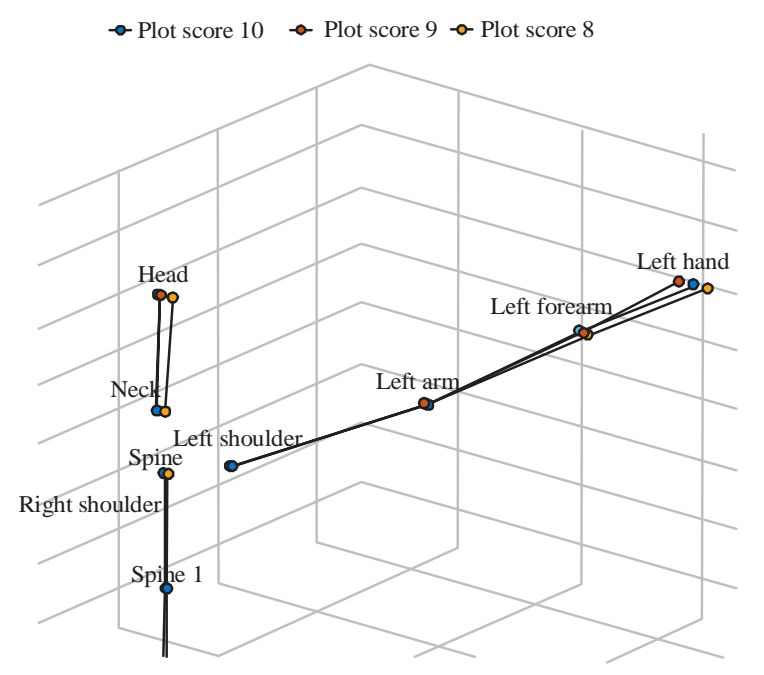

Fig. 6: Plotting the third archer's left hand

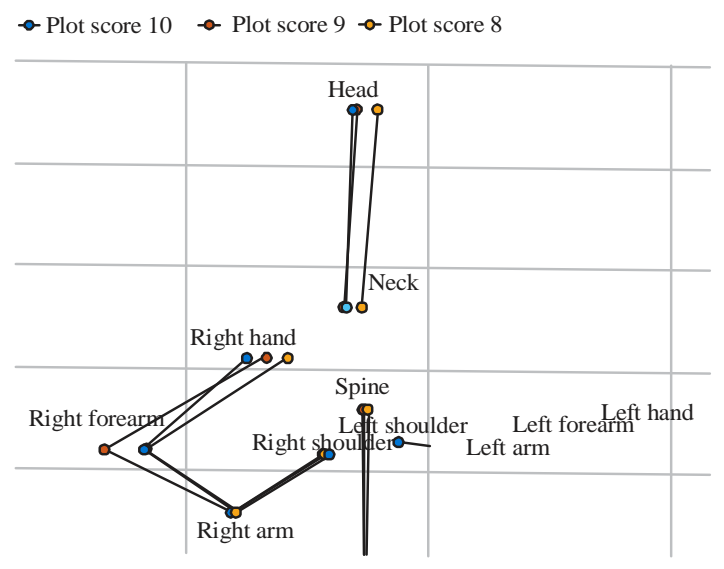

Fig. 7: Plotting the third archer's right hand

Figure 7 above is the same as Fig. 5 and 3 which is a figure of the archer's right hand part taken from the northeast of the archer. The color point in the figure is also a mapping of the third archer's right hand part that is connected to the blue line. It appears that there are only three different types of scores, so, there are only three in the figure. In Fig. 7 above, the distance of the right hand position score is 10 with the position of score 8 quite far even though the right forearm distance of both scores has a short distance. The right hand from score 10 tends to draw the right hand to the left after the shot while the position of the score of 8 directions forearm with right hand tends to be parallel.

\section{CONCLUSION}

The use of motion capture as a tool in the search for crucial points of a limb in archery can be done. The use of Manhattan distance distance calculations can also be done to find the deviation value of each shooting pose from the archer. By using the MATLAB program to process record data that has been previously converted from raw data in the Perception Neuron application into a database that is composed of Excel, so that, it can be further processed to find the deviation value per each pose and position of each each member of the body is crucial. In retrieval of data in this study, many data found invalid or could not be used due to the loss of the position of the sensor a certain part during the recording of the athlete's movements. Based on the testing in this study, many changes were found in the position of the right hand, left hand and foot of the archer. Based on the analysis that has been carried out, the first archer changes the score at the end of the session with the direction of the hand deviating to the right, as well as the second and third archers who sometimes drop scores at the end of several shots due to changes in the right hand direction where this right hand serves as a determinant of the direction of the shot.

\section{REFERENCES}

Adams, C., 2018. Six steps to improve bow accuracy. Rocky Mountain Elk Foundation Nonprofit Organization (RMEF), Wshington DC., USA. https://elknetwork.com/six-steps-improve-bowaccuracy/

Aprilia, Y.D., R. Latuconsina and T.W. Purboyo, 2018. A review of several algorithms for data mining. J. Eng. Appl. Sci., 13: 6157-6161.

Estevez-Garcia, R., J. Martin-Gutierrez, S.M. Mendoza, J.R. Marante, P. Chinea-Martin, O. Soto-Martin and M. Lodeiro-Santiago, 2015. Open data motion capture: MOCAP-ULL database. Procedia Comput. Sci., 75: 316-326.

Guest and Walls, J., 2017. 7 powerful archery tips for better accuracy (and a successful hunt). Average Hunter, USA. https://averagehunter.com/7-powerfularchery-tips-better-accuracy-successful-hunt/? doing_wp_cron=1537132966.4603788852691650390 625

Hammam, S.A., T.W. Purboyo and R.E. Saputra, 2017. Cotton texture segmentation based on image texture analysis using gray level run length and Euclidean distance. J. Theor. Appl. Inf. Technol., 95: 6915-6923.

Held, B., B. Moriarty and T. Richardson, 2018. Microsoft Excel Functions and Formulas. 4th Edn., Mercury Learning and Information, Dulles, Virginia, USA.,.

Khosla, G., N. Rajpal and J. Singh, 2014. Evaluation of euclidean and manhanttan metrics in content based image retrieval system. J. Eng. Res. Appl., 4: 43-49. 
Kolayis, I.E., M. Cilli, H. Ertan and J.A. Knicker, 2014. Assessment of target performance in archery. Procedia Soc. Behav. Sci., 152: 451-456.

Meredith, M. and S. Maddock, 2001. Motion capture file formats explained. MCS Thesis, University of Sheffield, Sheffield, England.

Ponnmoli, K.M. and S. Selvamuthukumaran, 2014. Analysis of face recognition using manhattan distance algorithm with image segmentation. Intl. J. Comput. Sci. Mobile Comput., 3: 18-27.

Sharma, A., M. Agarwal, A. Sharma and P. Dhuria, 2013. Motion capture process, techniques and applications. Intl. J. Recent Innov. Trends Comput. Commun., 1: 251-257.
Sinwar, D. and R. Kaushik, 2014. Study of Euclidean and Manhattan distance metrics using simple K-means clustering. Intl. J. Res. Appl. Sci. Eng. Technol., 2: $270-274$.

Umbrich, J., S. Neumaier and A. Polleres, 2015. Quality assessment and evolution of open data portals. Proceedings of the 2015 3rd International Conference on Future Internet of Things and Cloud, August 24-26, 2015, IEEE, Rome, Italy, pp: 404-411.

Vasquez, A., 2017. 8 health benefits of archery. World Archery Federation Sports Governing Body, Lausanne, Switzerland. https://worldarchery. org/news/147916/8-health-benefits-archery 